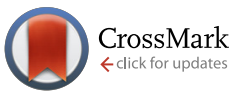

Cite this: Catal. Sci. Technol., 2015, 5,1668

\section{Developing catalytic materials for the oxidative coupling of methane through statistical analysis of literature data $\uparrow$}

\author{
Evgenii V. Kondratenko, ${ }^{\text {a }}$ Michael Schlüter, ${ }^{a}$ Manfred Baerns, ${ }^{b}$ David Linke ${ }^{a}$ \\ and Martin Holena*a
}

\begin{abstract}
Based on available 1870 literature data for the oxidative coupling of methane (OCM), various statistical models were applied i) to design three-component catalysts consisting of one host metal oxide $\left(\mathrm{La}_{2} \mathrm{O}_{3}\right.$ or $\mathrm{MgO}$ ) and two oxide ( $\mathrm{Li}, \mathrm{Na}, \mathrm{Cs}, \mathrm{Sr}, \mathrm{Ba}$, $\mathrm{La}$, or $\mathrm{Mn}$ ) dopants and ii) to predict their OCM performance. To validate this approach for catalyst design, selected materials were prepared and experimentally tested for their activity and selectivity in the target reaction. The effects of kinds of host oxides, dopants and their interplay on the OCM performance of differently composed catalysts were statistically evaluated.
\end{abstract}

Received 4th November 2014 Accepted 9th December 2014

DOI: 10.1039/c4cy01443j

www.rsc.org/catalysis ethane to ethylene: ethylene selectivity of 0.8 at around 0.7 ethane conversion. Under this consideration, the OCM reaction can become profitable when the ethylene selectivity would be close to the above value at a methane conversion higher than $0.30 .{ }^{14-16}$ The low selectivity to $\mathrm{C}_{2}$ hydrocarbons at industrially relevant degrees of methane conversion is related to higher reactivity of the target products tending to oxidation to $\mathrm{CO}$ and $\mathrm{CO}_{2}\left(\mathrm{CO}_{x}\right)$. To overcome such problems, methane can be converted into $\mathrm{C}_{2}$ hydrocarbons including acetylene in the absence of oxygen at temperatures above $2000{ }^{\circ} \mathrm{C}$ as had been previously utilized by Hüls AG and BASF. ${ }^{17}$ However, such process suffers from high energy demand. Hall reported about an alternative process concept for converting methane into ethylene and hydrogen or hydrocarbon liquids. ${ }^{18}$ In this concept, methane (less than $10 \%$ of the fed methane) is combusted in an internal-combustion thermal cracker where non-reacted methane is converted to mainly acetylene and hydrogen. The acetylene can then be hydrogenated with very high selectivity to ethylene using the hydrogen from the cracker. Recently, a dual-reactor concept was introduced for improving selectivity to higher hydrocarbons in the OCM reaction with a simultaneous decrease in $\mathrm{CO}_{x}$ selectivity. ${ }^{19,20}$ In this concept, the OCM reaction is performed in a first reactor, while a second reactor operating at a lower temperature and located downstream to the first one is used for hydrogenation of OCM-generated $\mathrm{CO}_{x}$ to hydrocarbons in presence of additional hydrogen. More than $50 \%$ of $\mathrm{CO}_{x}$ products formed in the OCM reactor were hydrogenated to desired $\mathrm{C}_{3}, \mathrm{C}_{4}$ and $\mathrm{C}_{5}$ hydrocarbons, with propane selectivity being up to 0.10 . An overall yield of $\mathrm{C}_{2+}$ hydrocarbons of 0.38 was achieved. Further progress of this concept can be expected when active and selective catalysts for $\mathrm{CO}_{x}$ hydrogenation will be developed. Alternatively, this concept 
or the OCM reaction itself would profit from new catalytic materials with improved OCM selectivity.

Some years ago, Thybaut et al. ${ }^{21}$ have suggested a methodology for catalyst design based on the kinetics of the OCM reaction. The idea behind their approach is to correlate catalytic performance with specific catalyst descriptors influencing individual reaction pathways. The following descriptors were defined and applied: i) reaction enthalpy of hydrogen abstraction from $\mathrm{CH}_{4}$, ii) chemisorption energy of $\mathrm{O}_{2}, \mathrm{CH}_{2} \mathrm{O}$, $\mathrm{HCO}, \mathrm{CO}, \mathrm{CO}_{2}, \mathrm{H}_{2} \mathrm{O}$, and $\mathrm{C}_{2} \mathrm{H}_{4}$, iii) initial sticking probabilities of $\mathrm{O}_{2}, \mathrm{CH}_{3}{ }^{\circ}, \mathrm{CO}, \mathrm{CO}_{2}$, and $\mathrm{H}_{2} \mathrm{O}$, and iv) concentration of active sites. By optimizing these parameters and reaction conditions, a yield of $\mathrm{C}_{2}$ hydrocarbons of 0.35 was predicted but not experimentally verified.

From a general viewpoint, the chemical composition of catalysts should influence such descriptors. Therefore, establishing relationships between the catalyst composition and OCM performance may open the possibility for designing effective catalytic materials. To this end, Zavyalova et al. ${ }^{22}$ have statistically analysed about 1000 scientific papers and patents reporting quantitative data on the chemical composition of OCM catalysts and their performance under different reaction conditions. These authors suggested a strategy for designing multicomponent catalysts; promising OCM catalysts should consists of host basic metal oxide ( $\mathrm{MgO}$ or $\mathrm{La}_{2} \mathrm{O}_{3}$ ) promoted with metal oxide dopant(s) influencing positively $\mathrm{C}_{2}$-selectivity (Cs, Na, Sr, Ba) and activity (Mn, W).

Unfortunately, no theoretically based relationship between catalyst composition and performance is known for the oxidative coupling of methane. Thus, such a relationship can only be established empirically, i.e. through fitting some mathematical function(s) to the available experimental data. The simplest kind of such empirical models is linear regression, which is however not suitable for this purpose because catalytic data typically do not reveal any linear dependence on catalyst composition. Consequently, quadratic or some special cubic models known as response surface have been traditionally used. ${ }^{23}$ Owing to the increasing power of modern computers, it became possible to use more sophisticated and complex nonlinear models, such as artificial neural networks, ${ }^{24}$ kernel regression, ${ }^{25}$ or Gaussian processes ${ }^{26}$ for catalyst research ${ }^{27-29}$ and for mathematical modelling in catalysis. ${ }^{30-32}$ Such empirical models can be trained on the basis of available catalytic data and hereafter used to predict performance of other catalysts that had not been used in the training. There are two typical purposes of such prediction approaches:

i) To check the trained model for its predictability, catalytic materials with compositions predefined by researchers are experimentally prepared and tested. Since only the catalysts from this predefined set are taken into account, it doesn't matter whether some of the available catalysts used for training the model have better performance than the catalysts from this set. However, some good-performing materials can be discovered because the composition of virtual catalysts is varied in a more systematic way compared to classical trial and error approach. ii) To find the best catalyst, the search, is performed in the whole considered set of catalyst compositions with the use of an optimization method, e.g. an evolutionary algorithm. ${ }^{25}$ When the model from (i) fits the experimental data well enough, it can be used for suggesting catalyst formulations in such evolutionary approach.

The main purpose of the present study was to experimentally validate the strategy for generating OCM catalysts based on host and dopant metal oxides as theoretically suggested in ref. 22. The specific objectives of the study were: (i) to elucidate the potential of statistical models for predicting OCM performance of multicomponent materials, (ii) to prove if there is a systematic difference in catalytic performance between materials possessing different main components, and (iii) to check if there is a synergy effect between two dopants. To this end, we applied various statistical models for suggesting potential catalyst compositions and tested their OCM performance experimentally. Finally, the models are critically evaluated in terms of their predictive accuracy.

\section{Theoretical approach and experimental details}

\section{Mathematical modelling}

From a mathematical viewpoint, eqn (1) represents an empirical model of a scalar performance feature, such as yield, selectivity or conversion as a function of catalyst composition or/and reaction conditions. Such function for a quadratic model is given by eqn (2). Other models considered in this paper are several variants of a particular kind of artificial neural networks, called radial basis function (RBF) networks. ${ }^{33}$ They are thoroughly explained in the electronic $\mathrm{ESI} \dagger$ together with the corresponding functions in eqn (1). Model parameters in artificial neural networks are called weights.

$$
\begin{gathered}
f: \mathscr{R}^{d+p} \rightarrow \mathscr{R}, \text { i.e. }, f(x, w) \in \mathscr{R}, \\
f(x, w)=w_{0}+\sum_{j=1}^{d}\left(w_{j} x_{j}+w_{j, j} x_{j}^{2}\right)+\sum_{j=2}^{d} \sum_{k=1}^{j-1} w_{j, k} x_{j} x_{k} .
\end{gathered}
$$

where $\mathscr{R}^{d+p}$ denotes the space of $(d+p)$-dimensional real vectors, $d$ is the dimension of input data $x$ (catalyst composition, reaction parameters ), and $p$ is the number of model parameters, i.e. the dimension of the vector of model parameters $w$. For a quadratic function $p=\frac{d(d+3)}{2}+1$ and $w=$ $\left(w_{0}, w_{1}, \ldots d, w_{1,1}, \ldots w_{1, d}, w_{2,1}, \ldots w_{d, d}\right)$.

The choice of a particular model kind, i.e. a particular definition of $f$ in eqn (1), is the most important modelling decision. However, it does not enable to make model predictions for a particular input $x$. To this end, a particular value $\hat{w}$ of the vector $w$ of parameters must also be chosen. The process of choosing $\hat{w}$ is called model learning or model training. For model learning, we need a set $\mathscr{M}$ of input data (in our case, a set of catalyst compositions) such that for each $x \in \mathscr{N}$, one or more measured values of the 
considered catalyst performance are available. The overall number of measurements available for $x$ is denoted $n(x)$, whereas the notation $m(x)=\left(y_{1}, \ldots, y_{n(x)}\right)$ is used for the whole sequence of the corresponding measured values. Most frequently, $n(x)$ is equal to 1 , but a model must be able to also deal with a situation that several different performance values have been measured for the same input $x$, e.g. in different experiments or by different laboratories. Each pair $(x, y)$, in which $x \in \mathscr{N}$ and $y$ is one of the corresponding values $y_{i}$ from the sequence $m(x)$, is called a training pair for the model. Mathematically, model learning is the minimization of some error $E$ with respect to its parameters using the available training pairs, thus the resulting $\hat{w}$ fulfils eqn (3),

$$
E(f, \widehat{w}, \mathscr{M})=\min _{w \in W} E(f, w, \mathscr{M}) .
$$

To solve the minimization task in eqn (3), two further decisions must be made:

- Within which set $W$ should the vector $w$ be sought? Frequently, $W$ is simply the space of all $p$-dimensional real vectors. Sometimes, however, some restrictions are imposed to some components of $w, e . g$., they have to be positive.

- How to compute the error $E(f, w, \mathscr{M})$ ? The kinds of errors most frequently used are the mean absolute error (MAE) and the mean squared error (MSE) defined in eqn (4) and (5), respectively.

$$
\begin{aligned}
& \operatorname{MAE}(f, w, \mathscr{M})=\frac{1}{\sum_{x \in \cdot \mathscr{M}} n(x)} \sum_{x \in \cdot \mathscr{M}} \sum_{\substack{i=1, m(x)=\left(y_{1}, \ldots, y_{n(x)}\right)}}^{n(x)}\left|f(x, w)-y_{i}\right|, \\
& \operatorname{MSE}(f, w, \mathscr{M}, \mathscr{M})=\frac{1}{\sum_{x \in \cdot \mathscr{M}} n(x)} \sum_{x \in \cdot \mathscr{M}} \sum_{\substack{i=1, m(x)=\left(y_{1}, \ldots, y_{n(x)}\right)}}^{n(x)}\left(f(x, w)-y_{i}\right)^{2} .
\end{aligned}
$$

Since the physical units of MAE and MSE are different, the root mean squared error (RMSE) is frequently used instead of MSE, which has the same units as MAE,

$$
\operatorname{RMSE}(f(x, w), \mathscr{\mu})=\sqrt{\operatorname{MSE}(f, w, \mathscr{\mu})}
$$

Model learning (eqn (3)) based on RMSE gives, of course, the same result as model learning based on MSE. In our research, we employed model learning based on RMSE, and for comparison, we also recorded MAE.

It is important to note that MAE, MSE and RMSE measure the error of the model but are not suitable to estimate the error of a particular prediction by the model because they do not include the information about the particular input for which the prediction is computed. Therefore, to estimate the error of a particular prediction in our experiments, we used a heuristics based on nearest neighbours, ${ }^{34,35}$ which proceeds in two steps:

1. Given the input $x_{\text {new }}$, in which the prediction $f\left(x_{\text {new }}, \hat{w}\right)$ has been computed, and a number $k$ chosen in advance, the elements of $\mathscr{N}$ are ordered increasingly according to their distance from $x_{\text {new }}$, and the average of the measured values $y^{(1)}, \ldots, y^{(k)}$ corresponding to the $k$ elements $x^{(1)}, \ldots, x^{(k)}$ of $\mathscr{M}$ closest to $x_{\text {new }}$ is computed, i.e. $\bar{y}^{(k)}=\frac{1}{k} \sum_{i=1}^{k} y^{(i)}$. For ordering the elements of $\mathscr{N}$, any distance can be used. When estimating the errors of predictions in our experiments, we used the Euclidean distance standardized with respect to different variance of proportions of different elements, which form the dimensions of the points in $\mathscr{N}$.

2. As an estimate of the prediction $f\left(x_{\text {new }}, \hat{w}\right)$, its distance from $\bar{y}^{(k)}$ is then taken, i.e. the value $\left|f\left(x_{\text {new }}, \hat{w}\right)-\bar{y}^{(k)}\right|$. According to step 1 , this distance depends on the choice of $k$. Therefore, we have always computed it for $k=2,3,4,5$ and used the maximum of those 4 values.

For modelling the dependence of yield of $\mathrm{C}_{2}$ hydrocarbons on composition of OCM catalysts, we used 2 different model types mentioned below. Their mathematical description is provided in the ESI. $\dagger$

I. The traditional quadratic response surface model described in eqn (2).

II. Clustered RBF network with diagonal Gaussian radial basis functions centred in data, optionally in addition combined with linear regression.

\section{Catalyst preparation}

$\mathrm{La}_{2} \mathrm{O}_{3}$ and $\mathrm{MgO}$ were used as host oxides for preparation of catalytic materials. Each host oxide was impregnated with an aqueous solution of two dopants at room temperature according to the incipient wetness impregnation method. The dopants were nitrates of lithium, sodium, caesium, barium, strontium and manganese. The impregnated materials were dried at $393 \mathrm{~K}$ for 4 hours followed by their calcination in a muffle furnace in static air at $1073 \mathrm{~K}$ for 5 hours.

\section{Catalytic testing}

Catalytic tests were carried out in a multi-channel reactor system at ambient pressure using a feed with methane and air in a stoichiometric $\mathrm{CH}_{4}: \mathrm{O}_{2}$ ratio of 2. This setup consists of 48 plug-flow fixed-bed quartz tube reactors (i.d. $=4 \mathrm{~mm}$ ) operating in parallel. $78 \mathrm{mg}$ of the fresh catalyst (sieve fraction of $250-450 \mu \mathrm{m}$ ) diluted with $\mathrm{SiC}$ in the ratio of $1 / 3$ were filled into each reactor. An in-house developed flow restrictor equally distributed the total gas flow to 48 reactors to ensure the modified contact time of $0.0039 \mathrm{~g} \mathrm{~min} \mathrm{ml}^{-1}$ per reactor. Before testing, the catalysts were heated up to $1073 \mathrm{~K}$ in a flow of air. Hereafter, the methane-air mixture was fed to the reactors. 
The feed components and the reaction products were analysed by an on-line gas chromatograph (Agilent 7890) equipped with PLOT/Q (for $\mathrm{CO}_{2}$ ) $\mathrm{AL} / \mathrm{S}$ (for hydrocarbons) and Molsieve 5 (for $\mathrm{H}_{2}, \mathrm{O}_{2}, \mathrm{~N}_{2}$, and $\mathrm{CO}$ ) columns as well as flame ionization and thermal conductivity detectors. The GC analysis started after the system reached reaction temperature and was carried out sequentially for each individual reactor. The $\mathrm{CH}_{4}$ conversion was calculated from the inlet and outlet molar flows of $\mathrm{CH}_{4}$. The product selectivity was calculated on feed basis.

\section{Results and discussion}

\section{Selection of catalyst compositions based on literature data}

For modelling, we defined 378 three-component catalyst formulations consisting of one host oxide $\left(\mathrm{La}_{2} \mathrm{O}_{3}\right.$ or $\left.\mathrm{MgO}\right)$ and two dopants from oxides of $\mathrm{Li}, \mathrm{Na}, \mathrm{Cs}, \mathrm{Sr}, \mathrm{Ba}, \mathrm{La}$, or Mn. For each host oxide, there were 21 individual groups differing in dopant combinations. In addition, each group consisted of 9 catalyst formulations based on individual dopant concentrations varying from 0.1 to $10 \mathrm{wt}$.\%. In order to estimate the yield of $\mathrm{C}_{2}$ hydrocarbons for these materials, we used various models of type (II) as defined in section "Mathematical modelling". In total, there were 13 models. All the models were trained using available literature data on catalysts and their OCM performance from ref. 22. The individual models differ from each other in:

i. The definition of the model described by eqn (1), in particular, in the number of clusters and of basic functions ( $c f$. ESI $\dagger$ ) used for modelling in each cluster,

ii. The details of the minimization procedure used to solve the optimization problem described by eqn (3),

iii. The data used for their training. Three subsets of the available literature data were considered:

A. 4 models for yield of $\mathrm{C}_{2}$ hydrocarbons were trained using catalysts based on host oxides of La or Mg (495 catalysts, 50 elements);

B. 4 models for yield of $\mathrm{C}_{2}$ hydrocarbons were trained using catalysts based on host oxides of La or $\mathrm{Mg}$ prepared via impregnation (181 catalysts, 31 elements);

C. 5 models for yield of $\mathrm{C}_{2}$ hydrocarbons were trained using catalysts containing only elements from the pool of oxides of $\mathrm{Mg}$, $\mathrm{La}, \mathrm{Sr}, \mathrm{Ba}, \mathrm{Na}, \mathrm{Cs}, \mathrm{Li}, \mathrm{Mn}$, and $\mathrm{W}$ (386 catalysts, 9 elements).

To compare the trained models with respect to their predictive accuracy, a 10-fold cross-validation on the considered subset of literature data was performed. Such subset was randomly divided into ten parts of approximately equal size. Hereafter, for each part, the RMSE of a model trained on the remaining 9 parts was computed and finally, the RMSE was averaged over all 10 parts. The two lowest average RMSE values were obtained for models trained on the subset $C$ of literature data. Therefore, only predictions made by models trained on the subset $C$ were finally taken into account for the decision which catalysts will be experimentally prepared and tested for the OCM reaction. To this end, a weighted average prediction of the yield of $\mathrm{C}_{2}$ hydrocarbons was calculated for each catalyst composition using the predictions by the five models trained on the subset $C$.

The weights of the 5 predictions were inversely proportional to the RMSE of each model. Within each catalyst group consisting of nine materials, the catalyst composition with the highest weighted average yield was synthesized. Table 1 reports these compositions and their predicted OCM performance. For clarity purposes, we classified the catalysts into 8 systems differing in host metals and promoters: $\mathrm{La}-\mathrm{Sr}$, $\mathrm{La}-\mathrm{Ba}, \mathrm{La}-\mathrm{Mg}$, La-alkali, $\mathrm{Mg}-\mathrm{Sr}, \mathrm{Mg}-\mathrm{Ba}, \mathrm{Mg}-\mathrm{La}$, and $\mathrm{Mg}-$ alkali. On average, catalysts based on $\mathrm{La}_{2} \mathrm{O}_{3}$ as host oxide should perform superior to their MgO-based counterparts. The highest average $\mathrm{C}_{2}$ yield of around 0.156 is predicted for

Table 1 Nominal composition of catalysts prepared on $\mathrm{La}_{2} \mathrm{O}_{3}$ and $\mathrm{MgO}$ basis. $Y_{\operatorname{Pr}}$ and $Y_{E x}$ stand for their predicted and experimental yields of $C_{2}$ hydrocarbons respectively

\begin{tabular}{|c|c|c|c|c|c|c|c|c|c|c|c|}
\hline \multirow[b]{2}{*}{ Nr. } & \multirow[b]{2}{*}{ Catalysts } & \multicolumn{8}{|c|}{$\begin{array}{l}\text { Metals and their weight } \\
\text { concentrations/wt.\% }\end{array}$} & \multirow[b]{2}{*}{$\mathrm{Y}_{\mathrm{Pr}}$} & \multirow[b]{2}{*}{$\mathrm{Y}_{\text {Ex }}$} \\
\hline & & $\mathrm{La}$ & $\mathrm{Mg}$ & $\mathrm{Sr}$ & $\mathrm{Ba}$ & $\mathrm{Li}$ & $\mathrm{Na}$ & Cs & Mn & & \\
\hline 1 & LaSrMn & 90.8 & & 9.1 & & & & & 0.1 & 0.148 & 0.141 \\
\hline 2 & LaSrBa & 90.8 & & 9.1 & 0.1 & & & & & 0.148 & 0.164 \\
\hline 3 & LaSrLi & 90.8 & & 9.1 & & 0.1 & & & & 0.139 & 0.173 \\
\hline 4 & LaSrNa & 90.8 & & 9.1 & & & 0.1 & & & 0.138 & 0.160 \\
\hline 5 & LaSrCs & 90.8 & & 9.1 & & & & 0.1 & & 0.148 & 0.147 \\
\hline 6 & LaBaMn & 98.9 & & & 1.0 & & & & 0.1 & 0.147 & 0.115 \\
\hline 7 & LaBaLi & 99.8 & & & 0.1 & 0.1 & & & & 0.135 & 0.154 \\
\hline 8 & LaBaNa & 90.8 & & & 0.1 & & 9.1 & & & 0.153 & 0.140 \\
\hline 9 & LaBaCs & 99.8 & & & 0.1 & & & 0.1 & & 0.146 & 0.149 \\
\hline 10 & LaMgMn & 90.8 & 9.1 & & & & & & 0.1 & 0.147 & 0.103 \\
\hline 11 & LaMgSr & 83.3 & 8.3 & 8.3 & & & & & & 0.151 & 0.148 \\
\hline 12 & LaMgBa & 90.1 & 9.0 & & 0.9 & & & & & 0.147 & 0.159 \\
\hline 13 & LaMgLi & 90.8 & 9.1 & & & 0.1 & & & & 0.134 & 0.141 \\
\hline 14 & LaMgNa & 90.8 & 9.1 & & & & 0.1 & & & 0.146 & 0.139 \\
\hline 15 & LaMgCs & 90.1 & 9.0 & & & & & 0.9 & & 0.146 & 0.127 \\
\hline 16 & LaLiMn & 83.3 & & & & 8.3 & & & 8.3 & 0.165 & 0.110 \\
\hline 17 & LaNaMn & 90.1 & & & & & 9.0 & & 0.9 & 0.155 & 0.137 \\
\hline 18 & LaCsMn & 83.3 & & & & & & 8.3 & 8.3 & 0.158 & 0.090 \\
\hline 19 & LaNaLi & 83.3 & & & & 8.3 & 8.3 & & & 0.156 & 0.130 \\
\hline 20 & LaNaCs & 90.8 & & & & & 9.1 & 0.1 & & 0.153 & 0.145 \\
\hline 21 & LaCsLi & 90.8 & & & & 9.1 & & 0.1 & & 0.147 & 0.147 \\
\hline 22 & MgSrMn & & 83.3 & 8.3 & & & & & & 0.141 & 0.009 \\
\hline 23 & MgSrBa & & 83.3 & 8.3 & 8.3 & & & & & 0.13 & 0.167 \\
\hline 24 & MgSrLi & & 83.3 & 8.3 & & 8.3 & & & & 0.138 & 0.102 \\
\hline 25 & MgSrNa & & 83.3 & 8.3 & & & 8.3 & & & 0.146 & 0.157 \\
\hline 26 & MgSrC & & 83.3 & 8.3 & & & & 8.3 & & 0. & 0.168 \\
\hline 27 & MgBaMn & & 90.8 & & 9.1 & & & & & 35 & 0.119 \\
\hline 28 & MgBaLi & & 90.8 & & 0.1 & 9.1 & & & & 0.145 & 0.062 \\
\hline 29 & $\mathrm{MgBa}$ & & 90.1 & & 0.9 & & 9.0 & & & 0.153 & 0.018 \\
\hline 30 & MgBaCs & & 83.3 & & 8.3 & & & 8.3 & & 0.128 & 0.153 \\
\hline 31 & MgLaMn & 9.1 & 90.8 & & & & & & & 46 & 0.088 \\
\hline 32 & MgLa & 8.3 & 83.3 & 8.3 & & & & & & 0.136 & 0.139 \\
\hline 33 & MgL & 9.0 & 90.1 & & 0.9 & & & & & 0.145 & 0.127 \\
\hline 34 & MgLaLi & 8.3 & 83.3 & & & 8.3 & & & & 0.151 & 0.104 \\
\hline 35 & MgLaNa & 8.3 & 83.3 & & & & 8.3 & & & 0.156 & 0.158 \\
\hline 36 & MgLaCs & 8.3 & 83.3 & & & & & 8.3 & & 0.138 & 0.120 \\
\hline 37 & MgLiMn & & 90.8 & & & 9.1 & & & & 0.145 & 0.097 \\
\hline 38 & MgNaMn & & 90.8 & & & & 9.1 & & & 0.154 & 0.010 \\
\hline 39 & MgCsMn & & 90.8 & & & & & 9.1 & & 0.135 & 0.093 \\
\hline 40 & MgNaLi & & 90.8 & & & 9.1 & 0.1 & & & 0.145 & 0.064 \\
\hline 41 & MgNaCs & & 90.8 & & & & 0.1 & 9.1 & & 0.135 & 0.112 \\
\hline 42 & MgCsLi & & 90.8 & & & 9.1 & & 0.1 & & 0.145 & 0.072 \\
\hline
\end{tabular}


La-Alkali system. The worst catalytic performance is predicted for catalysts from the $\mathrm{Mg}-\mathrm{Sr}$ group.

\section{Experimental catalytic data and their comparison with literature}

To check the contribution of non-catalytic methane oxidation to products distribution determined in presence of catalytic materials, we initially performed tests with an empty reactor and reactors filled with $\mathrm{SiC}, \mathrm{Al}_{2} \mathrm{O}_{3}$ or fused $\mathrm{SiO}_{2}$. In all cases, carbon oxides and $\mathrm{C}_{2}$ hydrocarbons were detected but methane conversion stayed below 0.025 and oxygen conversion below 0.04 . When catalytic materials were tested, conversion of methane and oxygen was significantly higher. Thus, the below results can be safely ascribed to the effect of catalyst on the OCM reaction.

Fig. 1 exemplarily illustrates the yield of $\mathrm{C}_{2}$ hydrocarbons experimentally determined over catalysts from the La-Sr and $\mathrm{Mg}-\mathrm{Sr}$ groups as a function of time on stream. The corresponding conversion of methane is given in Fig. S1 in the ESI. $\dagger$ The results obtained over catalysts from the La-Ba, La-Mg, La-alkali, $\mathrm{Mg}-\mathrm{Ba}, \mathrm{Mg}-\mathrm{La}$, and $\mathrm{Mg}$-alkali groups are summarized in Fig. S2-S7. For comparative purposes, the OCM performance of non-doped $\mathrm{La}_{2} \mathrm{O}_{3}$ and $\mathrm{MgO}$ is also presented in these figures. It should also be noted that not all catalytic materials showed stable OCM performance over $40 \mathrm{~h}$ at $1073 \mathrm{~K}$. Therefore, Table 1 reports initial yields of $\mathrm{C}_{2}$ hydrocarbons. One can clearly see that the yield is influenced both by host oxide and dopants. With the exception
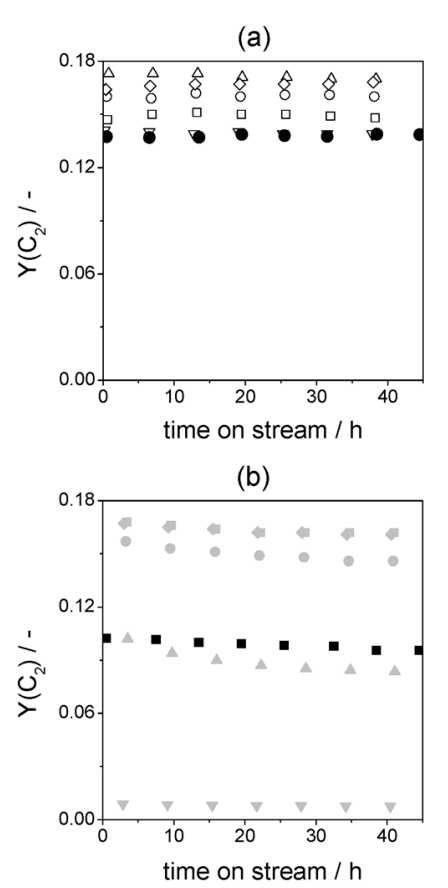

Fig. 1 Time on-stream yield of $\mathrm{C}_{2}$ hydrocarbons over (a) La-Sr-based $\left(\bullet-\mathrm{La}_{2} \mathrm{O}_{3}, \square\right.$-LaSrCs, O-LaSrNa, $\Delta$-LaSrLi, $\nabla$-LaSrMn, $\diamond$-LaSrBa) and (b) Mg-Sr-based ( $\square-\mathrm{MgO}, \square-\mathrm{MgSrCs}$, - $-\mathrm{MgSrNa}, \triangle-\mathrm{MgSrLi}, \nabla-\mathrm{MgSrMn}$, $-\mathrm{MgSrBa}$ ) catalysts at $1073 \mathrm{~K}$ and $\tau$ of $0.0039 \mathrm{~g} \mathrm{~min} \mathrm{ml}^{-1}$ using a feed consisting of $29 \% \mathrm{CH}_{4}$ in air. of MgBaLi, MgBaNa, MgNaLi, MgCsLi, oxygen conversion was complete over all catalysts tested.

Fig. 2 shows methane conversion and selectivity to $\mathrm{C}_{2}$ hydrocarbons experimentally obtained over catalysts synthesized in the present study together with the literature data available through ref. 22. From a viewpoint of yield of $\mathrm{C}_{2}$ hydrocarbons (used as criterion in our study for selecting catalytic materials), a visual inspection of all these figures suggests that the worst agreement between the previous literature and our present data was obtained for the $\mathrm{Mg}$-Alkali and $\mathrm{La}-\mathrm{Mg}$ systems, while the performance of other systems was reasonably predicted. To validate this interpretation statistically, we tested the equality of yield distributions between the literature data and our measurements using the 2-sample Kolmogorov-Smirnov test. ${ }^{36}$ Table 2 summarizes the achieved significance level for rejecting the hypothesis that the distributions of literature and experimental yields of $\mathrm{C}_{2}$ hydrocarbons are equal.

The achieved significance level is the probability that the obtained data can occur if the hypothesis is true, i.e. if both groups of data really follow the same distribution. If that probability is low, it is interpreted as the hypothesis being rejected by the test, and the lower the probability is, the higher is the significance of its rejection. For the La-Ba, La-alkali, La-Sr, Mg-Ba and $\mathrm{Mg}-\mathrm{Sr}$ systems, the tested hypothesis cannot be rejected. It can be rejected at the low significance level of $10 \%$ for the $\mathrm{Mg}$-La system, but not at the usual significance level of $5 \%$. For the $\mathrm{Mg}$-alkali and La-Mg systems, it can be rejected even at the high significance level of $1 \%$. Based on this statistical analysis, it can be concluded that our models realistically predict the OCM performance of differently composed catalysts with the exception of those from the $\mathrm{Mg}$-alkali and $\mathrm{La}-\mathrm{Mg}$ systems. It is beyond the current study to elucidate possible reasons for the latter deviation.

\section{Comparison between prediction and experiment}

To illustrate the predictive accuracy of our modelling approach, we calculated the difference between predicted and experimental yields of $\mathrm{C}_{2}$ hydrocarbons. The smaller the difference, the better the prediction is. If this difference has a negative value the modelling underestimates the experimentally determined OCM performance, while the situation is reverse when such value is positive. Fig. 3 compares the difference values for all catalysts. On average, the OCM performance of catalysts based on $\mathrm{La}_{2} \mathrm{O}_{3}$ is better predicted than that of their MgO-containing counterparts. The large difference for MgBaLi, MgBaNa, MgNaLi, MgCsLi is due to their low activity.

It is worth repeating that the predicted yields were obtained by using a model that is a weighted combination of five RBF network models trained with the subset $C$ of the available literature data. To check the predictive accuracy of individual models, we selected six particular models. Their selection was made according to the model predictive 


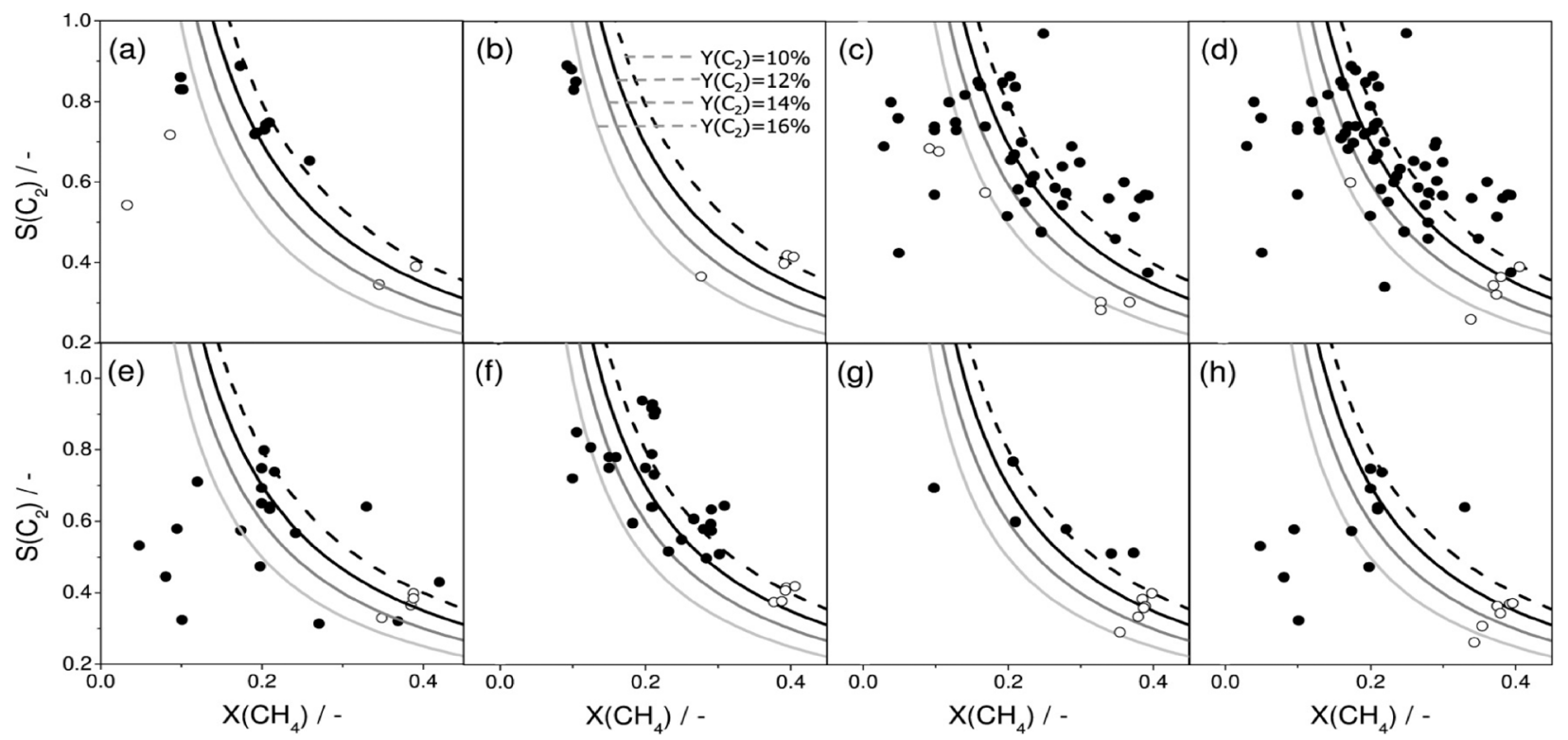

Fig. 2 Comparison between literature data (solid symbols) available through ref. 22 and data measured in the present study (open symbols) for the systems: (a) Mg-Ba, (b) Mg-Sr, (c) Mg-La, (d) Mg-Alkali, (e) La-Ba, (f) La-Sr, (g) La-Mg and (h) La-Akali. The experimental conditions are as in the caption of Fig. 1.

ability estimated by 10 -fold cross-validation. They include three models of type I (quadratic response surface models), which were trained with the subsets $A, B$ and $C$ of the literature data, respectively, and three models of type II (RBF network models) again trained with the subsets $A, B$ and $C$, respectively.

The MAE and RMSE values for each of the tested models are given in Table 3. It is important to note that, according to eqn (4) and (6), the lower these prediction errors, the better the predictive model accuracy is. Irrespective of the model applied, the lowest MAE and RMSE values were determined for the La-Ba system followed by the La-alkali, $\mathrm{Mg}$ - $\mathrm{La}$ and $\mathrm{Mg}$-alkali systems. The values, however, depend on the model and literature data subset used. When using the subset $C$, the lowest error was obtained nearly always with a model of type I. For all other subsets, models of type II appear to have better predictive accuracy. This model type also provided the lowest MAE and RMSE values when it was trained on the subset $A$.

To get insights into the errors in prediction of yield of $\mathrm{C}_{2}$ hydrocarbons, we checked the distribution of that error for

Table 2 Results of testing the equality of distributions between literature and experimental yields of $\mathrm{C}_{2}$ hydrocarbons using the 2-sample Kolmogorov-Smirnov test

\begin{tabular}{lc}
\hline System & Significance level/\% \\
\hline La-Ba & 30.0 \\
La-Alkali & 67.0 \\
La-Mg & 6.1 \\
La-Sr & 39.0 \\
Mg-La & 25.0 \\
Mg-Alkali & 0.2 \\
Mg-Ba & 68.0 \\
$M g-S r$ & 63.0
\end{tabular}

the catalysts tested in our experiments, as well as for their most important subgroups. Those subgroups were obtained using a regression tree ${ }^{37}$ for regression of the prediction error depending on the main component of the catalyst and on the presence of individual dopants. This tree is shown in Fig. 4 together with the distributions of prediction errors in the obtained subgroups. Analysing this tree, the following important influences on the prediction error on yield of $\mathrm{C}_{2}$ hydrocarbons were identified:

i. Most important feature from the point of view whether the prediction error is high or low is presence of $\mathrm{Mn}$ among dopants.

ii. Among catalysts not having $\mathrm{Mn}$ as a dopant, the next most important feature is the usage of $\mathrm{Sr}$ as a dopant.

iii. In all 3 subgroups resulting from the above two subdivisions, the next most important feature is always the main component, i.e. $\mathrm{La}_{2} \mathrm{O}_{3}$ or $\mathrm{MgO}$.

As seen from Fig. 4, the distributions of prediction errors for all tested catalysts is slightly shifted towards positive values (its mean value is 2.2 ) and is skewed to the right-hand side. Similar characteristics hold also for the specific subsets of catalysts containing neither $\mathrm{Mn}$ nor $\mathrm{Sr}$ as dopants or for catalysts containing $\mathrm{Mn}$ as a dopant, especially if $\mathrm{MgO}$ is the host oxide, though in those cases, they are based only on a rather low number of tested catalysts. On the other hand, the distribution of catalysts without $\mathrm{Mn}$ but containing $\mathrm{Sr}$ is slightly shifted towards negative values, though also this observation is based only on few catalysts.

The deviations between the predicted and the experimental values can originate from the facts that i) the models applied were inappropriate or ii) the literature data used to train the models strongly deviate from each other. To check the latter possibility, we analysed $\mathrm{C}_{2}$ hydrocarbons yields 


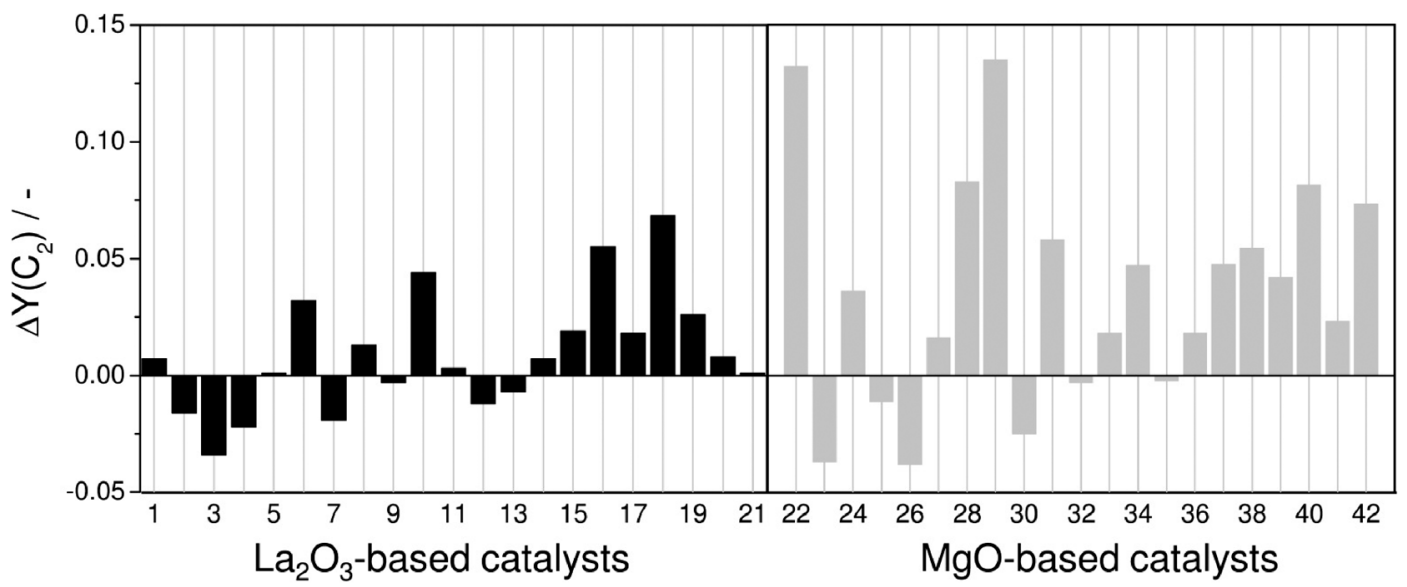

Fig. 3 Prediction error, i.e. the difference between predicted and experimental yield values of $C_{2}$ hydrocarbons $\left(\Delta Y C_{2}\right)$. The numbers of catalysts are explained in Table 1.

Table 3 Comparison of the MAE and RMSE errors with which different models predicted the yield of $\mathrm{C}_{2}$ hydrocarbons on various catalysts

\begin{tabular}{|c|c|c|c|c|c|c|c|c|c|}
\hline \multirow[b]{4}{*}{ Systems } & \multirow[b]{4}{*}{$E V(\mathscr{M})^{a}$} & & \multicolumn{7}{|c|}{ Best model of type I } \\
\hline & & & \multicolumn{2}{|l|}{$\underline{\text { Subset } A}$} & \multicolumn{2}{|c|}{$\underline{\text { Subset } B}$} & & \multicolumn{2}{|l|}{ Subset $C$} \\
\hline & & & \multicolumn{7}{|c|}{ Kind of model error } \\
\hline & & \multicolumn{2}{|r|}{ MAE } & RMSE & MAE & \multicolumn{2}{|c|}{ RMSE } & MAE & RMSE \\
\hline La-Ba & 5.6 & \multicolumn{2}{|r|}{2.6} & 3.2 & 3.5 & \multicolumn{2}{|c|}{3.9} & 1.4 & 1.6 \\
\hline La-alkali & 5.9 & \multicolumn{2}{|r|}{401.9} & 401.9 & 28.9 & \multicolumn{2}{|c|}{28.9} & 1.9 & 2.0 \\
\hline Mg-La & 3.6 & \multicolumn{2}{|r|}{2.6} & 2.8 & 2.1 & \multicolumn{2}{|c|}{2.5} & 2.6 & 3.2 \\
\hline \multirow[t]{5}{*}{ Mg-Alkali } & 3.9 & \multicolumn{2}{|r|}{6.9} & 7.3 & 6.5 & \multicolumn{2}{|c|}{6.9} & 6.9 & 7.0 \\
\hline & & \multicolumn{8}{|c|}{ Models of type II } \\
\hline & & \multicolumn{6}{|l|}{ Best } & \multicolumn{2}{|c|}{ Combined } \\
\hline & & \multicolumn{2}{|c|}{ Subset $A$} & \multicolumn{2}{|c|}{ Subset $B$} & \multicolumn{2}{|c|}{ Subset $C$} & & \\
\hline & & \multicolumn{4}{|c|}{$\overline{\text { Kind of model error }}$} & & & & \\
\hline Systems & $E V(\mathscr{M})^{a}$ & MAE & RMSE & MAE & RMSE & MAE & RMSE & MAE & RMSE \\
\hline $\mathrm{La}-\mathrm{Ba}$ & 5.6 & 0.6 & 0.7 & 1.4 & 1.8 & 1.6 & 2.6 & 1.7 & 2.0 \\
\hline La-Alkali & 5.9 & 1.6 & 2.0 & 2.5 & 3.2 & 1.9 & 2.6 & 1.9 & 2.7 \\
\hline $\mathrm{Mg}-\mathrm{La}$ & 3.6 & 2.4 & 2.7 & 2.5 & 2.3 & 2.8 & 3.4 & 2.4 & 3.2 \\
\hline Mg-Alkali & 3.9 & 4.9 & 5.2 & 5.0 & 6.0 & 8.5 & 8.8 & 8.1 & 8.5 \\
\hline
\end{tabular}

previously reported in literature for catalyst compositions, based on which the catalysts in the present study were prepared. The criteria for selecting literature data are summarized below:

i. $\mathrm{La}_{2} \mathrm{O}_{3}$ or $\mathrm{MgO}$ are host oxides with their weight percentages being between 83.3 and $99.8 \%$.

ii. Depending on the kind of catalytic system various dopants were considered as follows:

- For the La-Ba system: Ba, Na, Cs, Li, Mn;

- Ffor the La-Alkali system: Na, Cs, Li, Mn;

- For the Mg-La system: La, Sr, Ba, Na, Cs, Li, Mn;

- For the Mg-Alkali system: Na, Cs, Li, Mn.

The extent of variation $(E V(\mathscr{N}))$ of yield of $\mathrm{C}_{2}$ hydrocarbons in various literature studies was calculated according to eqn (7). In this equation, the set $\mathscr{N}$ for a certain catalytic system was selected according to the above criteria, $x$ and $y$ stand for catalyst composition and the corresponding yield respectively. For an ideal case, if the set of training pairs contains $\left(x_{i}, y_{i}\right)$ and $\left(x_{j}, y_{j}\right)$ and $x_{i}=x_{j}$ then $y_{i}$ should equal $y_{j}$. Inequality between $y_{i}$ and $y_{j}$ shows deviation between different published literature data.

$$
E V(\mathscr{M})=\frac{\sum_{\substack{x \in \mathscr{H}, n(x)>1, m(x)=\left(y_{1}, \ldots, y_{n(x)}\right)}} \sum_{i=2}^{n(x)} \sum_{j=2}^{i-1}\left|y_{i}-y_{j}\right|}{\sum_{\substack{x \in \mathcal{H} \\ n(x)>1}} \frac{n(x)(n(x)-1)}{2}}
$$

The $E V(\mathscr{M})$ values for available literature data are compared in Table 3 with the MAE and RMSE values showing the extent of deviation between experimentally determined and predicted yields of $\mathrm{C}_{2}$ hydrocarbons in the present study. From a general viewpoint, if the $E V(\mathscr{M})$ values are smaller 


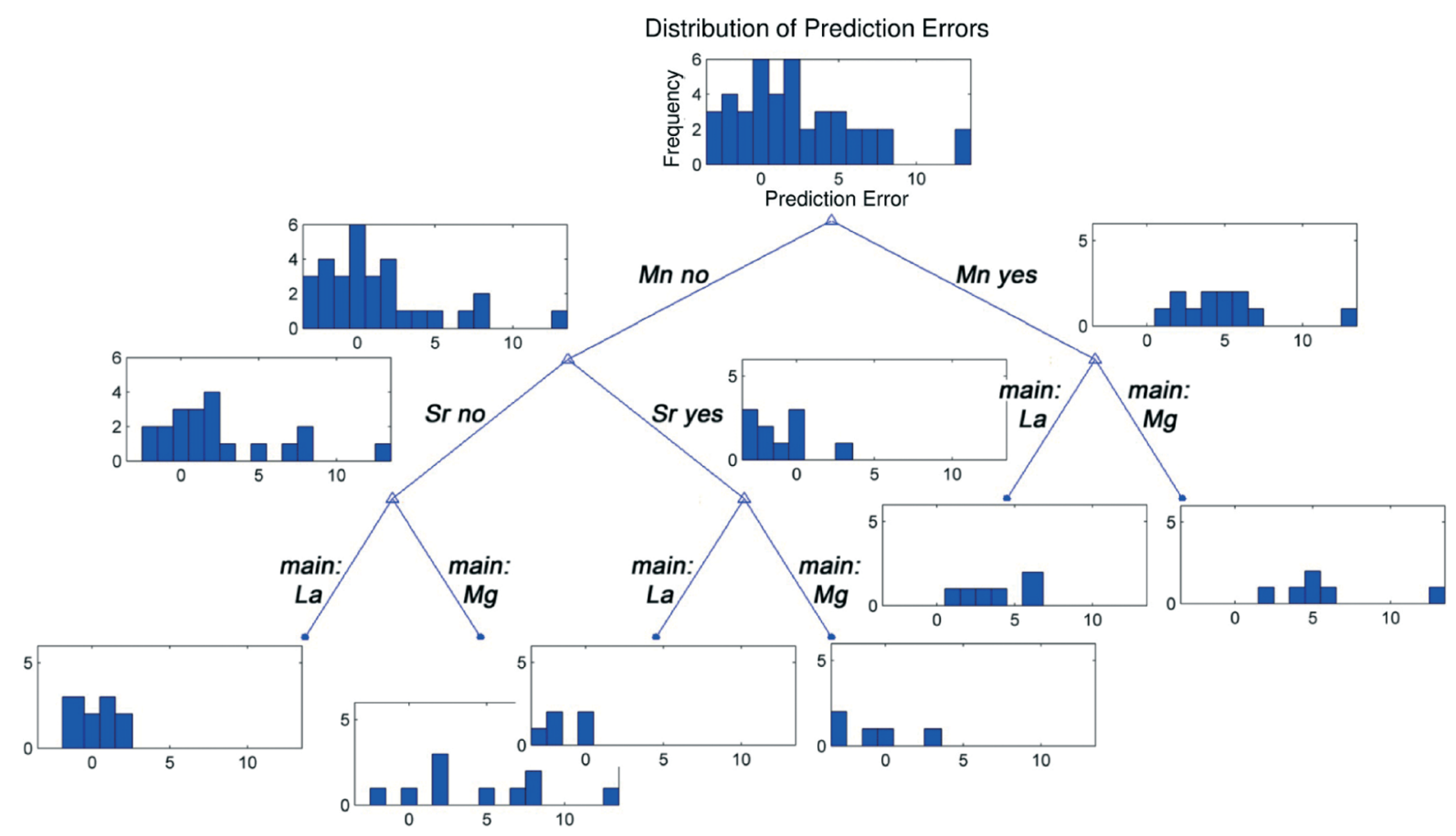

Fig. 4 Regression tree for the regression of the prediction error of yield of $C_{2}$ hydrocarbons (difference between the predicted and experimental yield) on the main component of the catalyst and on the presence of individual dopants. At each node and leaf of the tree, the distribution of the prediction error in the corresponding catalysts is shown.

than the errors (MAE or RMSE) between experimental and predicted values, then mainly the modelling approach is responsible for the problems with predictability. However, Table 3 shows that irrespective of the model applied, the MAE or RMSE values for the La-Ba and Mg-La systems are always smaller than $E V(\mathscr{M})$. In case of the La-Ba system, they are often substantially below (in particular, the error MAE of the RBF network trained with the subset $B$ of data is only about $10 \%$ of $E V(\mathscr{M}))$. For the La-Alkali system, the errors of all considered RBF networks are consistently below $E V(\mathscr{M})$, but among the traditional quadratic models, this is true only for the model trained with the $C$ subset of data, whereas the other two quadratic models are completely unusable.

In summary, at least the models of type II can be expected to correctly predict catalytic performance of materials based on the La-Ba, La-Alkali and Mg-La systems. This is not valid for the Mg-Alkali system, because the errors of all considered models are consistently larger than $E V(\mathscr{M})$.

\section{Relationships between catalyst composition and performance}

The purpose of the discussion below is to analyse possible relationships between the composition of three-component catalysts and their OCM performance. Particularly, we focus on the role of host oxide ( $\left.\mathrm{MgO} v s . \mathrm{La}_{2} \mathrm{O}_{3}\right)$ and dopants. In agreement with previous studies, ${ }^{38}$ pure $\mathrm{La}_{2} \mathrm{O}_{3}$ performed superior to $\mathrm{MgO}$ with respect to the yield of $\mathrm{C}_{2}$ hydrocarbons; 0.137 vs. 0.102 at complete conversion of oxygen. For our multicomponent catalysts, promoting $\mathrm{La}_{2} \mathrm{O}_{3}$ or $\mathrm{MgO}$ with $\mathrm{SrO}$ has the most positive effect on the yield; the highest $\mathrm{C}_{2}$ yields of 0.173 and 0.165 were achieved over LaSrLi and MgSrCs respectively (Table 1). According to the database in ref. 22 these values are actually higher than those reported in literature for catalysts based $\mathrm{La}_{2} \mathrm{O}_{3}$ or $\mathrm{MgO}$ as host components with the exception of $\mathrm{Li} / \mathrm{MgO}$ from ref. 39. This positive effect of SrO is valid for all tested catalysts irrespective of the second dopant used in our study, i.e., $\mathrm{Li}, \mathrm{Na}, \mathrm{Cs}, \mathrm{Ba}, \mathrm{Mg}$ and $\mathrm{Mn}$, with the last mentioned one showing a slightly negative effect. From a mechanistic viewpoint, promoting $\mathrm{La}_{2} \mathrm{O}_{3}$ with $\mathrm{SrO}$ results in the formation of anion vacancies in the lattice of the host oxide, ${ }^{40}$ which help to transform non-selective bi-atomic oxygen species on the catalyst surface into selective mono-atomic ones. ${ }^{41,42}$ When $\mathrm{BaO}$ was used as dopant, the yield of $\mathrm{C}_{2}$ hydrocarbons was also improved compared to pure $\mathrm{MgO}$ and $\mathrm{La}_{2} \mathrm{O}_{3}$. However, this is not valid for all combinations of $\mathrm{Ba}$ with another dopant. Moreover, Ba-promoted catalysts showed lower on-stream stability compared to the Sr-doped counterparts. This can be explained by the fact that barium oxide is transformed to the corresponding carbonate, which is more stable than strontium carbonate. For both $\mathrm{La}_{2} \mathrm{O}_{3}$ - and $\mathrm{MgO}-$ based catalysts, using alkali metal oxide as promoters also influenced this catalyst characteristics negatively.

The effect of individual dopants and dopant combinations on the yield of $\mathrm{C}_{2}$ hydrocarbons was further analysed by regression trees. The trees for the yield regression as a function of the dopants were constructed for the catalysts based on $\mathrm{La}_{2} \mathrm{O}_{3}$ and those based on $\mathrm{MgO}$, and they are shown in Fig. 5. The numbers in this figure stand for average $\mathrm{C}_{2}$ yields, with the value in the root being an average 


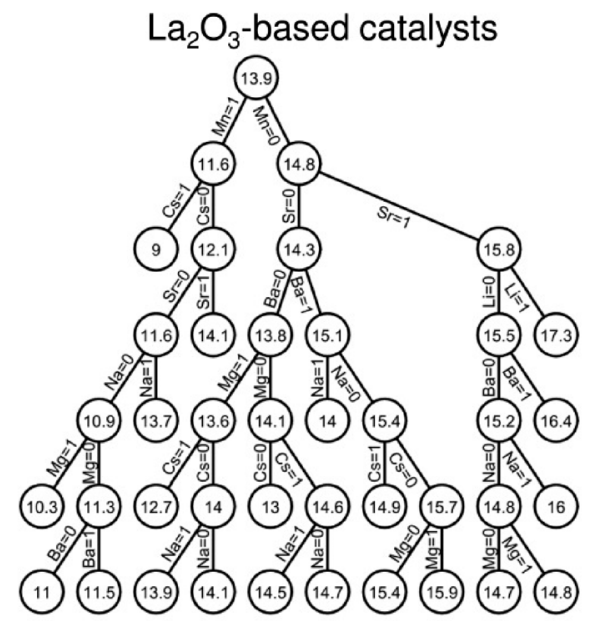

MgO-based catalysts

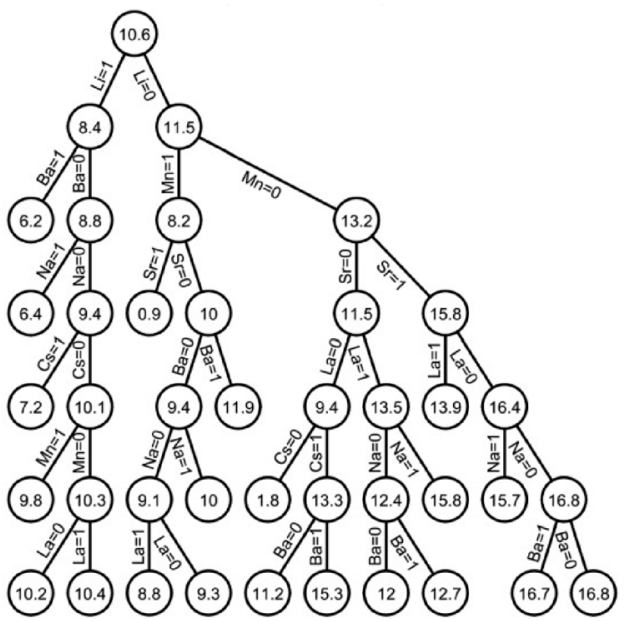

Fig. 5 Regression trees for the regression of yield (in percentage) of $C_{2}$ hydrocarbons over $\mathrm{La}_{2} \mathrm{O}_{3}$ - and $\mathrm{MgO}$-based catalysts on the presence of individual dopants. At each node and leaf of the tree, an average yield of the corresponding catalyst is given.

yield obtained over one of these two catalyst groups as a whole.

The paths from the root illustrate how the presence or absence of a certain dopant influences the yield. Among the catalysts from the $\mathrm{La}_{2} \mathrm{O}_{3}$ group, the presence of $\mathrm{Mn}$ has the most decisive influence on yield of $\mathrm{C}_{2}$ hydrocarbons. On average, this dopant negatively affects the yield. This statement contradicts to the theoretical conclusion from ref. 22 where Mn was identified as an important element positively influencing this catalyst property. This contradiction is probably due to the fact that $\mathrm{Mn}$ has been reported to play an important role as promoter only for supported catalysts possessing $\mathrm{Na}_{2} \mathrm{WO}_{4}$. Its positive effect on OCM performance was ascribed to the formation of catalytically active $\mathrm{Na}-\mathrm{O}-\mathrm{Mn}$ species ${ }^{43,44}$ and/or interconnected tetrahedral $\mathrm{WO}_{4}$ and octahedral $\mathrm{MnO}_{6}$ groups. ${ }^{45}$ Such catalyst compositions were not investigated in the present study. In the subgroup containing $\mathrm{Mn}$, the presence of $\mathrm{Cs}$ has the most decisive influence followed by the effect of Sr. The latter dopant also plays an important role in the subgroup free of $\mathrm{Mn}$. In the following leafs, the presence of $\mathrm{Li}$ and $\mathrm{Ba}$ is highly relevant for $\mathrm{C}_{2}$ hydrocarbons yield over catalysts without and with $\mathrm{Sr}$ respectively. However, the latter condition appears to be more important for achieving high yields.

The presence of Li has the most decisive influence on the yield of $\mathrm{C}_{2}$ hydrocarbons over catalysts based on $\mathrm{MgO}$ (Fig. 5). In the subgroup of Li-free materials, doping with $\mathrm{Mn}$ plays the second important role. The next important dopant influencing $\mathrm{C}_{2}$ yield is SrO. On average, catalysts containing this dopant showed the highest yield not only among MgObut also among $\mathrm{La}_{2} \mathrm{O}_{3}$-based catalysts.

Thus, taking the above statistically verified experimental results into account, we suppose that designing OCM catalysts based on one host metal oxide and two dopants may result in novel catalyst formulations with improved performance. Further progress can be expected when not only composition of catalysts but also their physico-chemical properties will be considered in statistical modelling. In addition, we strongly believe that supplementary information about the kinetics of individual reaction pathways should be taken into account in such approach to derive deeper fundamental relationships between catalyst composition and performance. We are currently performing such kinetic studies with the purpose to determine reaction constants of activation of $\mathrm{O}_{2}, \mathrm{CH}_{4}, \mathrm{C}_{2} \mathrm{H}_{6}$ and $\mathrm{C}_{2} \mathrm{H}_{4}$. Assuming that this statistical modeling turns out to be successful, evolutionary strategy in combination with high-through-put experimentation is to be applied for discovering optimal catalysts for maximal performance taking into account the multi-parameter space defined by the various variables (composition, solid-state properties, preparation methods, kinetic parameters and reaction conditions).

\section{Conclusions}

Statistical analysis of available literature data on OCM reaction was experimentally proven to have the potential for designing three-component catalysts based on one host metal oxide $\left(\mathrm{La}_{2} \mathrm{O}_{3}\right.$ or $\left.\mathrm{MgO}\right)$ and two dopants and for predicting their OCM performance. Catalysts prepared according to this approach performed superior to previously tested similarly composed materials. This analysis also enabled us to establish relationships between the catalyst composition and OCM performance. Compared with previous literature data, we cannot confirm that promoting with alkali metal oxides will always result in catalysts showing high yields of $\mathrm{C}_{2}$ hydrocarbons. To generate active and selective catalysts, it is important to combine such dopants with oxides of alkali-earth metals. Irrespective of the kind of second dopant, our study claims that $\mathrm{Sr}$ is the most promising dopant for $\mathrm{La}_{2} \mathrm{O}_{3}$ and $\mathrm{MgO}$.

From the point of view of modelling catalyst performance, our research has brought twofold experience:

1. Models based on RBF neural networks are more reliable than traditional quadratic response surface models.

2. The predictive accuracy of models based on literature data suffers from the high diversity of such data, which 
allows using only a part of the available information for model training, most naturally catalyst composition, whereas the remaining information, such as reaction conditions, is ignored and not incorporated into the model.

\section{Acknowledgements}

The development of some of the RBF network models employed in the reported research was partially funded by the Czech Science Foundation grant 13-17187S.

\section{Notes and references}

1 P. F. van den Oosterkamp, in Encyclopedia of Catalysis, ed. I. Horvath, John Wiley and Sons, New York, 2003, vol. 6, p. 456.

2 F. J. Keil, Microporous Mesoporous Mater., 1999, 29, 49.

3 M. Stocker, Microporous Mesoporous Mater., 1999, 29, 3.

4 G. F. Keller and M. M. Bhasin, J. Catal., 1982, 73, 9.

5 W. Hinsen and M. Baerns, Chem.-Ztg., 1983, 107, 223.

6 J. S. Lee and S. T. Oyama, Catal. Rev.: Sci. Eng., 1988, 30, 249.

7 J. H. Lunsford, Catal. Today, 1990, 6, 235.

8 M. Baerns and J. R. H. Ross, Catalytic Chemistry of Methane Conversion, Blackwell Scientific Publications, Oxford, 1992.

9 E. N. Voskresenskaya, V. G. Roguleva and A. G. Anshits, Catal. Rev.: Sci. Eng., 1995, 37, 101.

10 E. E. Wolf, Methane Conversion by Oxidative Process. Fundamentals and Engineering Aspects, Van Norstrand Reinhold, New York, 1992.

11 O. V. Krylov, Catal. Today, 1993, 18, 209.

12 V. D. Sokolovskii and E. A. Mamedov, Catal. Today, 1992, 14, 415.

13 E. V. Kondratenko and M. Baerns, in Handbook of Heterogeneous Catalysis, ed. G. Ertl, H. Knözinger, F. Schüth and J. Weitkamp, Wiley-VCH, Mannheim, 2008, vol. 6, p. 3010.

14 M. J. Gradassi and N. Wayne Green, Fuel Process. Technol., 1995, 42, 65.

15 J. H. B. J. Hoebink, H. M. Venderbosch, P. C. V. Geem, P. F. V. D. Oosterkamp and G. B. Marin, Chem. Eng. Technol., 1995, 18, 12.

16 T. Ren, M. K. Patel and K. Blok, Energy, 2008, 33, 817.

17 P. Pässler, W. Hefner, K. Buckl, H. Meinass, A. Meiswinkel, H.-J. Wernicke, G. Ebersberg, R. Müller, J. Bässler, H. Behringer and D. Mayer, Acetylene, in Ullmann's Encyclopedia of Industrial Chemistry, 2011, DOI: 10.1002/ 14356007.a01_097.pub4.

18 K. R. Hall, Catal. Today, 2005, 106, 243.

19 E. V. Kondratenko and U. Rodemerck, ChemCatChem, 2013, 5, 697.

20 M. Albrecht, U. Rodemerck and E. V. Kondratenko, Chem. Ing. Tech., 2014, 86, 1894.

21 J. W. Thybaut, J. Sun, L. Olivier, A. C. Van Veen, C. Mirodatos and G. B. Marin, Catal. Today, 2011, 159, 29.
22 U. Zavyalova, M. Holeňa, R. Schlögl and M. Baerns, ChemCatChem, 2011, 3, 1935.

23 R. H. Myers, D. C. Montgomery and C. M. Anderson-Cook, Response Surface Methodology: Proces and Product Optimization Using Designed Experiments, John Wiley and Sons, Hoboken, 2009.

24 S. Haykin, Neural Networks, A Comprehensive Foundation, IEEE Computer Society Press, New York, 1999.

25 B. Schölkopf and A. J. Smola, Learning with Kernels, MIT Press, Cambridge, 2002.

26 E. Rasmussen and C. Williams, Gaussian Process for Machine Learning, MIT Press, Cambridge, 2006.

27 M. Holeňa and M. Baerns, Catal. Today, 2003, 81, 485.

28 M. Moliner, J. M. Serra, A. Corma, E. Argente, S. Valero and V. Botti, Microporous Mesoporous Mater., 2005, 78, 73.

29 M. E. Günay and R. Yildirim, Chem. Eng. J., 2008, 140, 324.

30 J. M. Serra, L. A. Baumes, M. Moliner, P. Serna and A. Corma, Comb. Chem. High Throughput Screening, 2007, 10, 13.

31 S. C. Sieg, PhD Thesis, Modelling Quantitative Composition Activity Relationships (QCARs) for Heterogeneous Catalysts by Kriging and a Multilevel B-Spline Approach, Universität des Saarlandes, 2007.

32 M. Baerns and M. Holeňa, Combinatorial Development of Solid Catalytic Materials. Design of High-Throughput Experiments, Data Analysis, Data Mining, World Scientific - Imperial College Press, London, 2009.

33 M. D. Buhmann, Radial Basis Functions: Theory and Implementations, Cambridge University Press, Cambridge, 2003.

34 Z. Bosnić and I. Kononenko, Data Knowl. Eng., 2008, 67, 504.

35 S. Schaal and C. G. Atkeson, in Advances in Neural Information Processing Systems 6, Morgan Kaufmann Publishers, San Mateo, 1994, p. 160.

36 M. Neuhäuser, Nonparametric statistical tests. A computational approach, CRC Press, 2012.

37 L. Breiman, J. H. Friedman, R. A. Olshen and C. J. Stone, Classification and Regression Trees, Wadsworth, Belmont, 1984.

38 J. G. McCarty, A. B. McEven and M. A. Quinan, Stud. Surf. Sci. Catal., 1990, 55, 393.

39 T. Ito, J. Wang, C.-H. Lin and J. H. Lunsford, J. Am. Chem. Soc., 1985, 107, 5062.

40 H. Borchert and M. Baerns, J. Catal., 1997, 168, 315.

41 G. Gayko, D. Wolf, E. V. Kondratenko and M. Baerns, J. Catal., 1998, 178, 441.

42 E. V. Kondratenko, D. Wolf and M. Baerns, Catal. Lett., 1999, 58, 217.

43 D. Wang, M. P. Rosynek and J. H. Lunsford, J. Catal., 1995, 155, 390.

44 S. F. Ji, T. C. Xiao, S. B. Li, C. Z. Xu, R. L. Hou, K. S. Coleman and M. L. H. Green, Appl. Catal., A, 2002, 225, 271.

45 Y. Kou, B. Zhang, J. Z. Niu, S. B. Li, H. L. Wang, T. Tanaka and S. Yoshida, J. Catal., 1998, 173, 399. 